\title{
Philosophiques
}

Charrak, André. Empirisme et théorie de la connaissance. Réflexion et fondement des sciences au XVIII siècle, Paris, Vrin, 2009, 176 p.

\section{Vincent Guillin}

Volume 38, numéro 1, printemps 2011

URI : https://id.erudit.org/iderudit/1005733ar

DOI : https://doi.org/10.7202/1005733ar

Aller au sommaire du numéro

Éditeur(s)

Société de philosophie du Québec

ISSN

0316-2923 (imprimé)

1492-1391 (numérique)

Découvrir la revue

Citer ce compte rendu

Guillin, V. (2011). Compte rendu de [Charrak, André. Empirisme et théorie de la connaissance. Réflexion et fondement des sciences au XVIII ${ }^{\mathrm{e}}$ siècle, Paris, Vrin, 2009, 176 p.] Philosophiques, 38(1), 337-340. https://doi.org/10.7202/1005733ar d'utilisation que vous pouvez consulter en ligne.

https://apropos.erudit.org/fr/usagers/politique-dutilisation/ 


\section{Charrak, André. Empirisme et théorie de la connaissance. Réflexion et fondement des sciences au XVIII siècle, Paris, Vrin, 2009, 176 p.}

Avec Empirisme et théorie de la connaissance. Réflexion et fondement des sciences au XVIII siècle, André Charrak poursuit un itinéraire intellectuel qui, livre après livre, contribue à nous faire redécouvrir, en parcourant plus souvent les chemins de traverse d'une curiosité érudite que les voies royales d'une histoire des idées convenue, certains aspects de l'histoire de la philosophie classique et des Lumières aujourd'hui oubliés ou négligés. Faisant suite à une enquête sur les fondements de la théorie de l'harmonie (Raison et perception. Fonder l'harmonie au XVIII siècle; Paris, Vrin, 2001), à une analyse sur la réforme empiriste de la métaphysique opérée par Condillac (Empirisme et métaphysique. L'"Essai sur l'origine des connaissances humaines " de Condillac; Paris, Vrin, 2003), et à une étude sur la question de la modalité des lois de la nature dans l'empirisme des Lumières (Contingence et nécessité des lois de la nature au XVIII ${ }^{\mathrm{e}}$ siècle. La philosophie seconde des Lumières; Paris, Vrin, 2006), l'ouvrage dont il est ici rendu compte propose une perspective originale sur la tradition empiriste moderne qui, en s'appuyant sur ou en approfondissant explicitement certaines des orientations et des thèses antérieurement développées par l'auteur, en restitue à la fois la fécondité spéculative et la spécificité historique.

Originale, cette perspective l'est d'abord par les auteurs qu'elle aborde. $\mathrm{Si}$, avec Locke, on part bien d'un empiriste au pedigree irréprochable, André Charrak nous montre comment la thématique de la réflexion - comprise comme perception des opérations que l'esprit opère sur les idées reçues par les sens -, qui apparaît dans l'Essai concernant l'entendement humain, va être reprise et travaillée par une tradition empiriste «franco-berlinoise » à la lumière des critiques leibniziennes. C'est à partir de cette problématisation que les différentes virtualités gnoséologiques et métaphysiques dont la thématisation lockéenne de la réflexion était riche vont s'actualiser dans la pensée de Condillac ou de Rousseau. De la même manière, André Charrak, dans la deuxième partie de son ouvrage, montre comment la tentative empiriste de produire une architectonique des savoirs constitués (en réduisant les connaissances humaines à leurs supposés fondements dans l'expérience sensible) va se trouver confrontée à l'épineuse question du statut à accorder aux vérités mathématiques et à l'apodicticité que ces dernières semblent conférer aux lois de la mécanique rationnelle. C'est la prise en considération de ces problèmes qui va conduire D'Alembert et Maupertuis à reconnaître la spécificité des connaissances scientifiques et à essayer de proposer une reconstruction empiriste de leur constitution et de leur développement. Ainsi, et comme le lecteur s'en rendra vite compte, on aborde ici des auteurs qui, même si leur empirisme n'est pas contesté, comme Condillac, sont souvent les oubliés de l'histoire de cette tradition, ou des philosophes, comme Rousseau, dont la pensée s'élabore dans un dialogue critique avec l'empirisme. 
Pour le dire autrement, il y a bien une tradition empiriste continentale qui, tout en trouvant aussi dans Locke une de ses sources, a emprunté des voies différentes de celles de l'empirisme britannique classique.

Ces voies, et c'est là une autre originalité du travail d'André Charrak, sont très finement identifiées au moyen d'une approche qui articule élégamment recours à une doxographie des problèmes philosophiques et pratiques d'une analyse philologique qui en piste les modes de propagation effectifs dans la communauté savante. C'est ainsi que se trouve justifiée la thèse, jadis avancée par Cassirer à titre heuristique mais sans étayage textuel véritable, selon laquelle l'irruption et la diffusion dans le champ philosophique de la thématique de la réflexion, loin que d'être une conséquence du primat accordé au cogito dans la démarche cartésienne, trouvent leur origine dans le complexe d'idées formé par la découverte lockéenne du pouvoir propre de l'esprit à se connaître lui-même, et par les critiques adressées par Leibniz à cette "histoire naturelle de l'âme" qui se contentait à tort, selon lui, du phénomène psychologique alors qu'elle pourrait bien ouvrir la voie à une connaissance de la substance spirituelle et dont la tentative de retracer la genèse de nos idées était incapable de rendre raison de notre expérience de la nécessité. C'est bien cette lecture leibnizienne, nous montre - textes à l'appui - André Charrak, qui va faire surgir de l'indétermination de la position lockéenne les virtualités dont elle était grosse et qui, au travers d'un itinéraire passant, entre autres, par Wolff, Des Maizeaux, Helvétius et Quesnay, va aboutir à deux postures opposées mais qui se veulent aussi bien l'une que l'autre une réponse à cette même question "des limites qu'une enquête sur l'entendement humain doit assigner à la réflexion » (38). D'une part, avec Condillac, une radicalisation de cet «empirisme de la genèse » qui, poussant un cran plus loin le geste propre au way of ideas lockéen, va réduire toutes les opérations de l'âme (y compris la réflexion) à la seule sensation, ce qui du même coup fait s'évanouir la dimension d'activité propre à l'esprit, dont Leibniz tirait argument pour défendre sa position, et qui transforme la métaphysique en pure théorie de la connaissance. D'autre part, avec Rousseau, dont l'analyse psychologique entend poursuivre tout en la corrigeant celle de Condillac et qui ne s'effraye pas des conclusions ontologiques qu'on en pourrait tirer, une reconnaissance de la réflexion comme preuve de l'activité irréductible de l'esprit et dans laquelle on trouve "une révélation métaphysique irrécusable de la liberté de l'âme et, partant, de sa spiritualité » (82). On rejoint là encore une intuition de Cassirer qui interprétait le déploiement de la pensée de Rousseau comme «retour, qui lui est tout personnel, de Condillac à Leibniz ", mais que la démonstration lumineuse d'André Charrak permet dorénavant de "caractériser comme une réception [qui], loin d'être personnelle à Rousseau, spécifie l'empirisme franco-berlinois au siècle des Lumières" (87).

Une autre spécificité de cet empirisme franco-berlinois mise en évidence dans la seconde partie de l'ouvrage est le rapport spécifique qu'il 
entretient aux sciences positives. Comme nous l'avons évoqué précédemment, la thématique de la réflexion telle qu'elle se cristallise dans le bloc Locke-Leibniz soulève des questions relatives non seulement à la noèse (celle du statut de la réflexion comme opération), mais aussi aux noèmes, en particulier à ces "vérités de raison» mathématiques et scientifiques que leur universalité et leur nécessité semblent distinguer si nettement des "vérités de fait ». C'est ce second aspect de la critique leibnizienne que va reprendre à son compte l'empirisme continental en s'interrogeant sur la nature des propositions caractéristiques des savoirs constitués et sur les structures théoriques auxquelles elles appartiennent, à la différence de son homologue britannique qui s'intéressera surtout aux idées générales et abstraites. Ici encore, André Charrak expose avec une grande clarté la tension existant, à l'intérieur même de la branche continentale de la tradition empiriste, entre une adhésion de principe au projet de réduire les constructions scientifiques à leur fondement supposé dans la sensation et les réserves exprimées à l'égard des résultats atteints par les genèses effectivement tentées. Figure exemplaire de ce que l'on pourra tenir, en fonction de ses inclinations philosophiques, pour de la confusion conceptuelle ou de la vigilance critique, D’Alembert, qui, tout en accordant formellement que les idées mathématiques viennent de l'expérience, récuse les tentatives du théoricien de l'harmonie Rameau (dont l'importance cruciale pour la compréhension du projet réductionniste condillacien est à fort juste titre soulignée) ou du géomètre Clairaut pour reconduire les connaissances mathématiques à leur origine sensible.

Cette réticence de l'empirisme à pousser jusqu'à son terme son programme réductionniste, André Charrak s'emploie à nous convaincre qu'elle n'est pas la manifestation d'un quelconque scepticisme à l'égard de la connaissance scientifique, mais que, bien au contraire, elle ouvre la voie à une analyse empiriste originale, non plus des idées, mais des théories constituées, de leurs rapports et du rôle spécifique qu'y jouent les mathématiques. Prenant acte de l'effectuation de l'idéal classique de la mathesis universalis dans la réécriture algorithmique de la mécanique rationnelle newtonienne, les empiristes des Lumières vont dorénavant tenter de penser le fait de la mise en ordre des corpus scientifiques par l'outil mathématique, non plus sur le mode de la subalternation aristotélicienne ou de la science unitaire cartésienne, mais comme application de la rationalité mathématique à différents ordres de phénomènes, dont l'histoire ne se réduit plus à celle d'un esprit mais incorpore aussi celles des principes mis en œuvre. Cet «empirisme de la constitution ", qui prolonge ou accompagne l'" empirisme de la genèse ", on en trouve une bonne illustration dans l'Examen philosophique de la preuve de l'existence de Dieu donnée dans l' "Essai de cosmologie» (1756) de Maupertuis. Tout en sacrifiant à la tentative d'expliquer génétiquement la certitude mathématique au moyen d'une analyse psychologique, Maupertuis va en effet offrir dans cet écrit une «histoire raisonnée de l'éta- 
blissement des règles du choc ", qui, passant en revue les contributions successives de Descartes, Newton, Leibniz et Huygens, s'interroge sur la manière dont la nécessité apodictique des mathématiques "peut se communiquer entièrement à la mécanique, par le biais d'une application sans reste de la géométrie au phénomène du mouvement, dans laquelle on n'aurait à postuler aucune propriété extrinsèque au concept mathématique des corps » (146-147). C'est ce questionnement, conduit historiquement, sur «la connexion des sciences au plan de leurs fondements rationnels et des concepts qu'elles manipulent effectivement» (132) qui définit et singularise, nous dit André Charrak, la position de l'empirisme franco-berlinois en philosophie de la connaissance.

L'espace nous manque pour rendre justice à maintes analyses développées dans l'ouvrage, au premier rang desquelles l'examen très serré de l'hypothèse foucaldienne du lien entre mathesis et genèse qui conduit l'auteur à mettre en garde contre les dangers d'un usage non critique du concept d'épistémè en histoire de la philosophie. Qu'on nous permette seulement de conclure en saluant le souci constant qui l'anime de situer l'empirisme dans le fil d'une histoire qui ne le réduise pas à trouver son sens et son achèvement dans une «apothéose» kantienne et qui permet à André Charrak d'en restituer la diversité, l'originalité et la fécondité.

VINCENT GUILLIN

Université du Québec à Montréal

\section{Antognazza, Maria Rosa. Leibniz: An Intellectual Biography, Cambridge, Cambridge University Press, 2009, 623 p.}

Le livre de Maria Rosa Antognazza est une contribution accomplie non seulement aux études leibniziennes, mais également à l'histoire intellectuelle moderne en général. Son succès principal est à trouver dans sa manière de relever le défi le plus intimidant des études leibniziennes: l'immense diversité, parfois démesurée, des intérêts intellectuels de Leibniz qui, jusqu'à récemment, avait «disloqué et divisé» (p. 3) le corpus leibnizien aux yeux des spécialistes, sans compter qu'elle obscurcissait notre image de l'homme derrière l'œuvre. En se basant sur le corpus toujours croissant d'études choisissant une cohérence à la fois temporelle et thématique dans l'œuvre leibnizienne, et notamment sur l'ouvrage de Christia Mercer (Leibniz's Metaphysics: Its Origins and Development, Cambridge, 2001), la combinaison adroite par Antognazza de récits historiques fluides et d'analyses textuelles pertinentes vient satisfaire le besoin d'une analyse globale - particulièrement nécessaire pour les lecteurs anglophones -, de la vie et de l'œuvre du savant allemand itinérant.

Dans son introduction, Antognazza souligne les quatre thèses méthodologiques qui guident son analyse. D'abord, loin d'être réduites à des pré- 